\title{
EFECTIVIDAD DEL GASTO EN SALUD PÚBLICA Y SU IMPACTO SOBRE LA MORTALIDAD POR INFECCIÓN RESPIRATORIA AGUDA - COLOMBIA (2017)
}

\begin{abstract}
EFICÁCIA DAS DESPESAS COM A SAÚDE PÚBLICA E SEU IMPACTO NA MORTALIDADE DE INFECÇÃO RESPIRATÓRIA AGUDA - COLÔMBIA (2017).
\end{abstract}

EFFECTIVENESS OF PUBLIC HEALTH EXPENDITURE AND ITS IMPACT ON ACUTE RESPIRATORY INFECTION MORTALITY - COLOMBIA (2017) 


\section{Resumen}

Los programas de inmunización representan una de las principales actividades de Salud Pública (SP). OBJETIVO: Evaluar la efectividad del gasto en SP en función de la Mortalidad por Infección Respiratoria Aguda (IRA) y de la Cobertura de Programas de inmunización con Pentavalente, tercera dosis (menores de un año) en Colombia durante el año 2017. MATERIALES Y MÉTODOS: Estudio ecológico - transversal, de tipo analítico acerca del comportamiento de las variables en mención. RESULTADOS: No se evidencian correlaciones significativas entre el gasto ejecutado, y la mortalidad asociada a IRA, tampoco para con las coberturas en programas de inmunización. CONCLUSIÓN: Existen factores de confusión asociados a las variables de interés.

\section{Palabras claves: Salud} Pública,Medicina Preventiva, Programas de Inmunización, Costo y análisis de costos

\section{Abstract}

Immunization is one of the different activities developed by Public Health (PH). OBJECTIVE: To evaluate the effectiveness of $\mathrm{PH}$ expenditure based on Mortality from Acute Respiratory Infection (ARI) and Vaccination Coverage with Pentavalent, third dose (children less than one year old) in Colombia during 2017. MATERIALS AND METHODS: Study ecological - cross-sectional, analytical type about the course of the mentioned variables. RESULTS: There are no significant correlations between the expenditure executed, and the mortality associated with ARF, neither for the vaccination coverage. CONCLUSION: There are confounding factors associated with the variables of interest.

Keywords: Public Health, Preventive Medicine, Immunization Programs, Cost and cost analysis 


\section{INTRODUCCIÓN}

En general, la Salud Pública (SP)

se orienta hacia todas aquellas acciones tendientes a la prevención de la enfermedad, prolongar la vida, y promover la salud mediante el esfuerzo mancomunado de la sociedad $(1 ; 2)$; de allí que entre las múltiples funciones y fines de la SP, se destaque la de evaluar la efectividad, accesibilidad, y calidad de la prestación y administración de los servicios de salud (3). En este orden de ideas, y en el marco de la normatividad derivada del Plan Decenal de Salud Pública (PDSP) 2012 2021, y de la Estrategia PASE a la Equidad en Salud (4), la vida saludable y el control de las enfermedades transmisibles es una de las ocho dimensiones prioritarias de la SP en Colombia (4), las cuales deben ser sujeto de seguimiento y evaluación. Si bien se sabe que las acciones de SP e intervenciones colectivas se financian a partir de la Subcuenta de Salud Pública Colectiva ${ }^{1}$ (la cual se nutre de diferentes fuentes de recursos estatales), en una coyuntura donde el gasto total en SP para 2016 correspondió a 325 mil millones de pesos colombianos (5), cuyo monto se mantiene en promedio invariable entre 2012 y 2016 , y que representa aproximadamente el $0.02 \%$ del gasto total mensual en salud (equivalentes a poco más de 1500 millones de pesos en pesos colombianos corrientes de 2016; casi \$USD 465 millones); son pocos los estudios que correlacionen la inversión en SP (puntualmente programas de inmunización con Pentavalente para menores de 1 año, durante 2017); tampoco existen resultados que relacionen indicadores de mortalidad por infección respiratoria aguda (IRA) en menores de 1 año, con los otros indicadores: mortalidad asociada y porcentaje de cobertura de programas de inmunización por departamento.

Según documentos técnicos del Ministerio de Salud y Protección Social (6), entre 2012 y 2016 se optimizó la descentralización de recursos para la Salud Pública, confiando el criterio y la gobernanza de esta en los despachos de Alcaldes y Gobernadores, con la asesoría de sus respectivos secretarios de salud, y de las instituciones territoriales respectivas (direcciones seccionales de salud); en este periodo de tiempo el gasto en salud correspondió a 1,4 billones de pesos, manteniendo estables desde el gobierno central los costos de funcionamiento, con fluctuaciones en la inversión

\footnotetext{
${ }^{1}$ Los recursos destinados al Plan de Intervenciones Colectivas (PIC), como se designa técnicamente a las actividades de salud pública, se financian en forma solidaria y mancomunada (de acuerdo con la Ley 1438 de 2011) (COLOMBIA, Congreso de la República, 2011), se derivan de los fondos y subcuentas: Sistema General de Participaciones (SGP), fondos de recursos de las PES- y EPS-C para promoción y prevención, Subcuenta del ADRES de Promoción de la salud y Seguros de Riesgos Catastróficos, recursos de la Subcuenta para Accidentes de Tránsito (SOAT) dedicados a Promoción y Prevención (P\&P), recursos de las Administradoras de Riesgos Laborales (ARL) para actividades de (P\&P), recursos adicionales derivados de las Cajas de Compensación Familiar destinados también a actividades de (P\&P) en el marco de la estrategia nacional de Atención Primaria en Salud (APS).
} 
en 'SP (de $\pm 1.75 \%$ del total del gasto en salud; media: $22.8 \%)^{(7)}$.

Según datos del Banco de la República de Colombia, entre 2007 y 2013 el mayor porcentaje del gasto departamental en salud se ha concentrado en la atención de la población pobre no asegurada, con vigencias que fluctúan entre el 45 y el $56 \%$ del total del presupuesto nacional para la salud, siendo la SP el tercer ítem del gasto después de "otros gastos", y seguido por el gasto destinado al Régimen Subsidiado (8).

Por su parte, de los 10 proyectos de SP con mayor inversión del Ministerio de Salud y Protección Social (MSPS) durante el año 2016, la promoción de actividades de programas de inmunización en todo el territorio nacional representa el $42 \%$ del total del gasto, siendo así el proyecto de inversión en SP más importante para el MSPS (ver Figura No: 1).

Se hace relevante indagar respecto del comportamiento y la efectividad de la inversión en SP y en las actividades de programas de inmunización, toda vez esta actividad es fundamental para el logro del Objetivo número 3 de los Objetivos del Desarrollo Sostenible (ODS), (propuesto por la Organización de Naciones Unidas - ONU), el cual se propone "Garantizar una vida sana y promover el bienestar de todos a todas las edades", a través de (entre otros) "Lograr la cobertura sanitaria universal, incluida la protección contra los riesgos financieros, el acceso a servicios de salud esenciales de calidad y el acceso a medicamentos y vacunas inocuos, eficaces, asequibles y de calidad para todos" (9).

Ante la anterior coyuntura, este trabajo académico busca determinar la existencia de relaciones de dependencia entre las variables: Tasa de Mortalidad por infección respiratoria aguda (IRA) en menores de 1 año [X1], y el Grado de ejecución presupuestal de la Subcuenta de Salud Pública [X2], ambas en función de la Cobertura de programas de inmunización con Pentavalente a menores de un año de edad [Y1], reportados en el año 2017.

\section{MÉTODO}

Estudio ecológico - transversal, de tipo analítico, derivado del análisis estadístico del comportamiento de las variables: Tasa de Mortalidad por infección respiratoria aguda (IRA) en menores de 1 año [X1], y el Grado de ejecución presupuestal de la Subcuenta de Salud Pública [X2], ambas en función de la Cobertura de programas de inmunización con Pentavalente a menores de un año de edad [Y1], reportados en el año 2017 en el Formato Único Territorial (FUT) y 

GASTO EN SALUD PÚBLICA Y SU IMPACTO SOBRE LA MORTALIDAD POR INFECCIÓN RESPIRATORIA AGUDA

en el Sistema de información y consulta de distribuciones de recursos territoriales (SICODIS) provistos por el Departamento Nacional de Planeación de Colombia (DNP).

\section{Limitaciones del estudio}

Se hizo énfasis en los datos registrados para el año 2017 (y no por ejemplo tendencias por cohortes de tiempo), debido a limitaciones para obtener matrices de datos más recientes en las bases de datos públicas del gobierno Colombiano (esto a falta de datos desagregados tanto para el año en cuestión, así como para series temporales).

Otra importante limitación hace referencia al énfasis puesto en el indicador: Mortalidad por IRA (como variable independiente), la cual excluye de considerar otras variables de confusión que podrían determinar la efectividad del gasto y de la inmunización como actividad de SP (como por ejemplo: estado de salud física del menor, las variaciones en la morbimortalidad por IRA de acuerdo a los picos epidemiológicos, la frecuencia de inmunización en los territorios, las barreras geográficas, y la disponibilidad de instalaciones de salud para su realización).

\section{RESULTADOS}

Se identificó que para el año 2017 el Estado Colombiano invirtió trescientos veinte y cinco mil millones de pesos colombianos (equivalentes a USD\$ 98'136.512,50) en acciones de SP, donde la mayor concentración de la inversión se concentra en la capital del país (Bogotá D.C.), Antioquia, Valle del Cauca, y algunos de los departamentos de la región Caribe (Bolívar, Atlántico y Córdoba), aspecto que podría denotar dificultades en la descentralización de las actividades de SP; este comportamiento puede identificarse en la Figura 2.

Respecto a las coberturas y a la mortalidad asociada de los 33 departamentos analizados para el año 2017, la mayoría alcanzan coberturas superiores al $88 \%$ (con excepción del Caquetá, Amazonas, Guaviare, Vichada y Guainía); al observar las mayores tasas de Mortalidad por IRA, estas se agrupan en la capital y en los departamentos con algunas de las mayores coberturas de programas de inmunización; sin embargo, podría ser este un hallazgo incidental asociado a una mayor capacidad de notificación y registro de casos de IRA en los departamentos más centrales (Bogotá, Antioquia, Valle del cauca), y a aquellas regiones donde sus condiciones geográficas y climáticas predisponen a mayores casos de IRA (como ocurre en el Chocó 
por ejemplo), tal y como se aprecia en la Figura 3.

Al calcular el valor de $\mathrm{R}$ (coeficiente de correlación) para el modelo predictivo que involucra las variables a asociar: Mortalidad por IRA en menores de un año y Cobertura de Programas de inmunización con Pentavalente, se evidencia un valor de $p=0.44$ ( $p>0.05)$ evidenciando no significancia, así se descarta la hipótesis de trabajo (que proponía una asociación entre la Cobertura de programas de inmunización y la Tasa de Mortalidad asociada).

Al buscar la correlación entre el Gasto en Salud Pública (medido en términos de la ejecución presupuestal de la subcuenta de Salud Pública en 2017), y la Cobertura de Programas de inmunización con Pentavalente (a nivel territorial para 2017), se identificó un Coeficiente de Pearson (que da cuenta de la magnitud de la correlación entre ambas variables), señala un valor de 0.72 ( $p>0.05)$; lo que también confirma la no significancia, y descarta que la cobertura de Programas de inmunización con Pentavalente (tres dosis) menores de un año de edad este directamente relacionada con el porcentaje o grado de ejecución presupuestal en SP.

Con los datos sistematizados se procedió a graficar la dispersión para las variables: Mortalidad por IRA y Cobertura de Programas de inmunización con Pentavalente Menor a 1 Año 3a. Dosis, donde se identifican muchos casos de mortalidad que se alejan de la media de coberturas de programas de inmunización en los territorios, lo cual demuestra la no correlación, y postula la teoría de que existen otras variables de confusión determinantes de la mortalidad por IRA en los territorios, que poco tienen que ver con la programas de inmunización como actividad de protección específica, tal y como se aprecia en la Figura 4.

Se resalta el caso de algunos departamentos que demuestran las mayores ejecuciones presupuestales (Huila [VTPE: \$USD 1'967.755,98], Bogotá D.C. [VTPE: 10'423.077,78 \$USD], Antioquia [VTPE: 870.483,54 \$USD], y Valle del Cauca [VTPE: 7'172.097,35 $\$ \cup S D]$, pero que indistintamente del gasto demuestran unas Coberturas de Inmunización $(\mathrm{Cl})$ que fluctúan aleatoriamente entre altas y bajas: (Huila $[\mathrm{Cl}$ : 89.5\%], Bogotá D.C. [Cl: 89.86\%], Antioquia [Cl: $89.61 \%]$ y Valle del Cauca [Cl: $94.33 \%]$ ), como puede evidenciarse en la Tabla 1, y en la Figura 5.

En últimas, resulta evidente que existen zonas del país con una alta concentración de la inversión en actividades de la SP para algunos Departamentos, donde existen desigualdades entre aquellos correspondientes a la región central 

GASTO EN SALUD PÚBLICA Y SU IMPACTO SOBRE LA MORTALIDAD POR INFECCIÓN RESPIRATORIA AGUDA

en comparación con los territorios geográficamente más distanciados (periferia), escenarios donde existen las mayores barreras geográficas de acceso a los servicios de salud. Además, es allí donde existen limitaciones logísticas y operativas para la implementación de las actividades específicas de salud pública, normalmente circunscritas a la Atención Primaria en Salud / Salud Comunitaria (entiéndase los servicios intra y extramurales de programas de inmunización a la población en edad preescolar).

Se destaca además que existen variables de confusión, imposibles de rastrear desde los sistemas de indicadores anclados a las bases de datos públicas del DNP y del Ministerio de Salud, que condicionan tanto el comportamiento de la mortalidad asociada, así como de los porcentajes de coberturas en programas de inmunización.

\section{DIscusióN}

La Tasa de Mortalidad por IRA en Colombia, según cifras de la ONG Así Vamos en Salud, entre 1998 y 2008 viene presentando un retroceso progresivo, solamente interrumpido un leve incremento de 2011 a 2014, y un posterior declive hasta llegar a la cifra notificada en 2019, de 4,78 (ver Figura 6). Se destacan como factores coadyuvantes de dicha reducción (entre otras) el impacto de la política nacional anti-tabaquismo, el control de factores de riesgo (como son la exposición a elementos causantes de alergia), y el manejo médico de otros factores agravantes como son enfermedades de base (principalmente aquellas bronco-obstructivas) ${ }^{(11) .}$

El descenso señalado en la anterior figura demuestra que las acciones de saneamiento ambiental, higienización de entornos comunes, así como la vigilancia epidemiológica basada en el trabajo cooperativo interinstitucional, y control con acciones de prevención temprana (mediante lavado e higienización de las manos, uso de tapabocas), y de protección específica (principalmente inmunización en los grupos vulnerables de primera infancia y adultez mayor) tienen un efecto positivo directo sobre la ocurrencia de muertes asociadas a IRA (11); este tipo de intervenciones colectivas son genéricamente realizadas, dentro de las funciones esenciales de la salud pública en la mayoría de los países de Europa, y del hemisferio occidental. De otro lado, aunque las infecciones respiratorias agudas (IRA) en su mayoría de casos no constituyen la causa principal de muerte, si pueden mediar como desencadenantes o como comorbilidades asociadas a infecciones de la vía respiratoria que ponen en riesgo la vida $(12,13,14)$. 
La oferta de vacunas existentes en el mercado (tanto las de virus vivos, así como las inactivadas), han demostrado una efectividad que oscila entre el 70 y el $90 \%$ no solamente en los casos sintomáticos, sino también en personas sanas. Pese a lo anterior, los esfuerzos tendientes hacia la inmunización universal de los grupos poblacionales sigue siendo un reto para los sistemas de salud (15), especialmente debido a barreras geográficas, socio-políticas, culturales, y por divergencias en el manejo clínico de los síntomas; se destacan los aportes que el país por medio de las instituciones de salud y las aseguradoras que han llevado a mejorar la oportunidad y la distribución de los biológicos entre la población objeto (16).

Así, dado que las rinofaringitis (o resfriado común), constituyen una de las causas más comunes de consulta médica, esta en la mayoría de sus presentaciones es causada por distintos tipos de virus (rinovirus, coronavirus, adenovirus, y parainfluenza); este tipo de infecciones representa el $50 \%$ de las infecciones de las vías respiratorias superiores (17); su prevención, diagnóstico y tratamiento temprano resultan prioritarios, toda vez que minimizan los costos de atenciones de mayor complejidad asociadas a procesos patológicos como: Bronquiolitis, Neumonía, Crup laríngeo; y a largo pla- zo, se pueden asociar a Asma (principalmente por exposición a Virus Sincitial Respiratorio - VSR) (18).

Dada la naturaleza adaptativa que subyace a la replicabilidad de los virus, se hace difícil para la industria farmacéutica desarrollar alternativas de vacunas de tipo multivalente que afronten los distintos serotipos conocidos (por ejemplo) para los rinovirus (20). A este respecto los desarrollos sobre vacunas específicas para VSR y para el Virus de Parainfluenza tipo 3 (VPI3), se asocian también al descenso de la mortalidad asociada a IRA (21).

Aunque las vacunas para VSR y VPI3 (cada una), han demostrado disminuir los nuevos casos de IRA, sus complicaciones, y sus costos asociados por prolongaciones de las atenciones en salud, y por estancias hospitalarias, no están ampliamente documentados los fundamentos teórico - conceptuales, así como los criterios económicos, logísticos y técnicos asociados a la estructuración de campañas, estrategias y modelos de gestión para la programas de inmunización masiva preventiva de IRA desde una estrategia de Atención Primaria en Salud (APS) (21).

Un estudio ecológico desarrollado en el contexto colombiano en 2018, evidenció la influencia que ejercen las desigualdades socioeconómicas existentes 

GASTO EN SALUD PÚBLICA Y SU IMPACTO SOBRE LA MORTALIDAD POR INFECCIÓN RESPIRATORIA AGUDA

para niños y niñas en edad preescolar sobre la morbi-mortalidad por IRA, destacándose que contextos altamente marcados por deprivación socioeconómica (o pobreza extrema) incrementan el riesgo asociado de mortalidad en menores pertenecientes al decil correspondiente al estrato más bajo de la estructura social (22).

Existen otros factores condicionantes del impacto y grados de desarrollo de las estrategias de programas de inmunización contra IRA, entre las que se destacan: percepción del equipo de salud sobre el biológico (vacuna), percepción del usuario de servicios de salud sobre las estrategias y calidades del equipo de programas de inmunización (intra y extramural); y la presión económica que generan las vacunas entendidas como nuevas tecnologías, en unos sistemas de salud con limitaciones y vulnerabilidades presupuestales (21).

En un estudio de cohortes desarrollado en Argentina a partir de un modelo estadístico, se logró identificar que la programas de inmunización antigripal en niños entre 6 y 23 meses de edad, logró disminuir las tasas brutas de mortalidad en este grupo poblacional después de la programas de inmunización, incidiendo en una disminución de hasta 3 años del riesgo de morir por IRA. A pesar de la influencia del invierno so- bre los casos nuevos de IRA, se estima que la estrategia nacional de programas de inmunización antigripal en Argentina reduce en 3,5 casos de muertes al mes por dicha entidad patológica (23).

Todos estos factores asociados justifican la posible existencia de variables de confusión no identificadas por los instrumentos gerenciales usados por el Estado Colombiano para la aprobación y distribución de recursos públicos con destinación a las intervenciones colectivas de salud; esto explica parcialmente la no existencia de correlación explícita entre la Mortalidad por IRA y Cobertura de Programas de inmunización con Pentavalente (en menores de un año, 3ra dosis), así como para la ejecución del gasto de la subcuenta de Salud Pública asociada a las coberturas de programas de inmunización con Pentavalente para el mismo grupo poblacional.

Si bien la evidencia es sólida en demostrar la efectividad e impacto de la programas de inmunización contra IRA, como estrategia de prevención de la enfermedad (aunada a otras estrategias de promoción de la salud, aseguramiento de condiciones materiales de vida óptimas, y de desarrollo de ambientes, barrios y ciudades saludables), existe un reto para la Salud Pública, la Epidemiología de Campo, y la gestión de la APS en cuanto a la documentación, estudio, descripción 

GASTO EN SALUD PÚBLICA Y SU IMPACTO SOBRE LA MORTALIDAD POR INFECCIÓN RESPIRATORIA AGUDA

y posibilidad de predicción mediante modelos econométricos y bioestadísticos, que permitan armonizar y alinear la política pública en SP; la ejecución de recursos financieros de gasto y de inversión; la toma de decisiones sobre intervenciones colectivas de salud; así como el desarrollo de formas de trabajo solidario en red entre agentes hospitalarios, comunidades y equipos extramurales del primer nivel de atención, que redimensionen los modelos de gestión de costoefectividad para la gerencia de las actividades en SP.

\section{CONCLUSIÓN}

Existe en Colombia una creciente y significativa inversión pública en actividades de SP; la alta concentración del gasto en ciudades capitales y en departamentos de la región andina y caribe problematizan respecto al progreso de la gestión de una SP descentralizada.

No existe una correlación positiva fuerte entre la Mortalidad por IRA, y las coberturas de programas de inmunización, así como tampoco para la ejecución de recursos para la SP, y el eventual grado de cobertura de programas de inmunización con Pentavalente en menores de 1 año - tres dosis en total; pese a esta ausencia de evidencia, se resalta la vital importancia que tiene la programas de inmunización como principal estrate- gia de prevención de las enfermedades infectocontagiosas virales de la vía aérea. Existen variables de confusión (no contempladas por los instrumentos de gerencia de la salud pública), que determinan indirectamente la mortalidad por IRA.

\section{AgRAdeCIMIENTO}

Los autores del presente trabajo queremos agradecer el apoyo representado en asesorías temáticas y metodológicas, provistas por la Doctora María Luisa Latorre Castro MD. Esp. PhD, investigadora de la Escuela de Medicina Proyecto Maestría en Salud Pública, Fundación Universitaria Juan N. Corpas, por su valioso y siempre constante apoyo. Este estudio se desarrolló con recursos propios de la FUJNC. Los datos utilizados son de uso público, no requieren de especial autorización, solamente de su correcta referenciación y citación. 


\section{BIBLIOGRAFÍA}

1. Beaglehole, R.; \& Bonita, R. Public health at the crossroads. 1ra Ed. Cambridge: Cambridge University Press, 1997. ISBN: 0-521-58373-X.

2. Guest, C.; Riccardi, W.; Kawachi, I.; \& Lang, I (Ed.). Oxford Handbook of Public Health Practice. 3 Ed. Oxford, UK: Oxford University Press, Oxford Medical Publications, 2013. 656 p. ISBN: 978-019-958630-1

3. U.S. Department of Health and Human Services, Centers for Disease Control and Prevention (CDC). Introduction to Public Health: Public Health 101 Series. Atlanta, GA: Centers for Disease Control and Prevention (CDC), U.S. Department of Health and Human Services, 2015. [Internet]; Disponible en: https://www.cdc.gov/publichealth101/ documents/introduction-to-public-health.pdf Acceso: 04 oct. 2019.

4. Colombia, Ministerio de Salud y Protección Social. Plan Decenal de Salud Pública PDSP 2012-2021. Estrategia PASE a la Equidad en Salud: LINEAMIENTOS CONCEPTUALES. Bogotá D.C.: Dirección de Epidemiología y Demografía, Sistema de Monitoreo y Evaluación, Análisis de Situación de Salud, Ministerio de Salud y Protección Social; Fondo de Población de las Naciones Unidas (UNFPA); \& Universidad Externado de Colombia, 2014. [Internet]; 216 p. Disponible en: https:// www.minsalud.gov.co/sites/rid/Lists/BibliotecaDigi$\mathrm{t}$ a I / R I D E / V S / E D / P S P / Estrategia\%20PASE\%20A\%20LA\%20EQUIDAD\% 20EN\%20SALUD\%20-\%20Guia\%20Conceptual.pdf Acceso: 04 oct. 2019.

5. Colombia, Ministerio de Hacienda, Departamento Nacional de Planeación (DNP). Sistema de información y consulta de distribuciones de recursos territoriales - SICODIS; 01 pantalla. [Internet]; Disponible en: https://sicodis.dnp.gov.co/ReportesSGP/ReporteComparativo.aspx Acceso: 04 oct. 2019.

6. Colombia, Congreso de la República. Ley 1438 de 2011 "Por medio de la cual se reforma el Sistema General de Seguridad Social en Salud y se dictan otras disposiciones" (enero 19). Bogotá D.C.: Congreso de la República. 2011; Diario Oficial No.: 47957 de Enero 19 de 2011. [Internet]; 51 p. Disponible en: https://www.minsalud.gov.co/Normatividad_Nuevo/LEY\%201438\%20DE\%202011.pdf Acceso: 04 oct. 2019.

7. Arciniegas B, J.A.; Gómez L, D.E.; Suárez A, L.Y.; \& Cruz V, M.F. Estructura del Gasto en Salud Pública en Colombia. Bogotá D.C.: República de Colombia, Ministerio de Salud y Protección Social, Oficina Asesora de Planeación y Estudios Sectoriales - Grupo de Estudios y de Evaluación de Políticas Públicas. 2018; Papeles en Salud No.: 17. (Noviembre). (sin año). [Internet]; 46 p. Disponible en: https://www.minsalud.gov.co/sites/rid/
Lists/BibliotecaDigital/RIDE/DE/PES/estructuragasto-salud-publica-colombia.pdf Acceso: 04 oct. 2019.

8. Orózco-Gallo, A. J. Un análisis del gasto público en salud de los entes territoriales colombianos. Cartagena de Indias: Banco de la República Sucursal Cartagena. 2015. [Internet]; Serie Documentos de Trabajo sobre Economía Regional (220). 69 p. Disponible en: https://www.banrep.gov.co/sites/default/files/publicaciones/archivos/ dtser_220.pdf Acceso: 04 oct 2019.

9. Comisión Económica para América Latina y el Caribe (CEPAL); \& Organización de Naciones Unidas (ONU). La Agenda 2030 y los Objetivos de Desarrollo Sostenible: una oportunidad para América Latina y el Caribe. Santiago de Chile: ONU \& Publicaciones CEPAL, División de Publicaciones y Servicios Web. 2018; (LC/G.2681-P/Rev.3), [Internet]; 93 p. Disponible en: https://repositorio.cepal.org/bitstream/handle/11362/40155/24/ S1801141_es.pdf Acceso: 04 oct. 2019.

10.Así Vamos en Salud. Indicadores de Salud: Tasa de mortalidad por infección respiratoria aguda (IRA) en menores de 5 años - georreferenciado. Bogotá D.C.: Así Vamos en Salud. Octubre 25 de 2019. [Internet]; 01 pantalla. Disponible en: https:// www.asivamosensalud.org/indicadores/enfermedades-transmisibles/tasa-de-mortalidad-por-infeccion-respiratoria-aguda-ira-en Acceso: 04 oct. 2019.

11.García R, R.M. Factores de riesgo de morbilidad y mortalidad por infecciones respiratorias agudas en niños menores de 5 años. Rev. Med. Electrón. 2010. [Internet]; 32(3): 01 pantalla. Disponible en: h t t p://scielo.sld.cu/scielo.php? script=sci_arttext\&pid=S1684-1824201000030001 0 Acceso: 04 oct 2019.

12.Cox, M.; Rose, L.; Kalua, K.; de Wildt, G.; Bailey, R.; \& Hart, J. The prevalence and risk factors for acute respiratory infections in children aged 0-59 months in rural Malawi: A cross-sectional study. Influenza Other Respir Viruses. 2017. [Internet]; 11(6):489-496. doi:10.1111/irv.12481 Disponible en: https://onlinelibrary.wiley.com/doi/full/10.1111/ irv.12481 Acceso: 10 ene. 2020.

13.Reyes, H.; Perez-Cuevas, R.; Salmeron, J.; Tome, P.; Guiscafre, H.; \& Gutierrez, G.; Infant mortality due to acute respiratory infections: the influence of primary care processes. Health Policy Plan. 1997. [Internet]; 12(3): 214-223. doi:10.1093/heapol/ 12.3.214 Disponible en: https://academic.oup.com/ heapol/article/12/3/214/740045 Acceso: 10 ene. 2020.

14.Anteneh, Z.A.; \& Hassen, H.Y. Determinants of Acute Respiratory Infection Among Children in Ethiopia: A Multilevel Analysis from Ethiopian Demographic and Health Survey. Int J Gen Med. 2020. [Internet]; 13: 17-26. Disponible en: https:// 
www.ncbi.nlm.nih.gov/pmc/articles/PMC6996624/ pdf/ijgm-13-17.pdf Acceso: 10 ene. 2020.

15.Webster, R; Plotkin, S; \& Dodet, B. Emergence and Control of Viral Respiratory Diseases. Emerg Infect Dis. 2005. [Internet]; 11(4): e4. Disponible en: https://www.ncbi.nlm.nih.gov/pmc/articles/PMC3320327/ Acceso: 04 oct. 2019.

16.Greenberg, H.B.; \& Piedra, P.A. Immunization Against Viral Respiratory Disease: A Review. The Pediatric Infectious Disease Journal. 2004. [Internet]; 23(11): S254-S261. Disponible en: DOI: 10.1097/01.inf.0000144756.69887.f8 Disponible en: https://journals.Iww.com/pidj/Fulltext/ 2004/11001/Immunization_Against_Viral_Respiratory_Disease_A.11.aspx Acceso: 04 oct. 2019.

17.Bayona O, Y.; \& Niederbacher V, J. Infecciones respiratorias virales en pediatría: generalidades sobre fisiopatogenia, diagnóstico y algunos desenlaces clínicos. MÉD. UIS. 2015. [Internet]; 28(1): 133-141. Disponible en: http://www.scielo.org.co/ pdf/muis/v28n1/v28n1a14.pdf Acceso: 04 oct. 2019.

18.Osterhaus, A.; \& de Vries, P. Vaccination against acute respiratory virus infections and measles in man. Immunobiology. 1992. [Internet]; 184(2-3): 180-92. Disponible en: https://www.sciencedirect.com/science/article/pii/S0171298511804742? via\%3Dihub Acceso: 04 oct. 2019.

19.Murphy, B.R.; Hall, S.L.; Kulkarni, A.B.; Crowe, J.E Jr.; Collins, P.L.; Connors, M; Karron, R.A.; \& Chanock, R.M. An update on approaches to the development of respiratory syncytial virus (RSV) and parainfluenza virus type 3 (PIV3) vaccines. Virus Res. 1994. [Internet]; 32(1): 13-36. Disponible en: https://www.sciencedirect.com/science/
article/abs/pii/0168170294900590?via\%3Dihub Acceso: 04 oct. 2019.

20.Anderson, A.J.; Snelling, T.L.; Moore, H.C.; \& Blyth, C.C. Advances in Vaccines to Prevent Viral Respiratory Illnesses in Children. Paediatr Drugs. 2017. [Internet]; 19(6): 523-531. Disponible en: https://link.springer.com/article/10.1007\%2Fs40272-017-0257-x Acceso: 04 oct. 2019.

21.Nair, H.; Verma, V.R.; Theodoratou, E.; Zgaga, L.; Huda, T.; Simões, E.; Wright, P.F.; Rudan, I.; \& Campbell, $\mathrm{H}$. An evaluation of the emerging interventions against Respiratory Syncytial Virus (RSV)-associated acute lower respiratory infections in children. BMC Public Health, 2011. [Internet]; 13; 11 Supl 3:S30. Disponible en: https:// bmcpublichealth.biomedcentral.com/articles/ 10.1186/1471-2458-11-S3-S30 Acceso: 04 oct. 2019.

22.Alvis Z, N.J.; Castañeda O, C.; Díaz, D.P.; et al. Desigualdades en la mortalidad por infección respiratoria aguda en niños: un análisis colombiano. Biomédica. 2018. [Internet]; 38(4):586-593. doi: 10.7705/biomedica.v38i4.4062. Disponible en: https://www.revistabiomedica.org/index.php/biomedica/article/view/4062 Acceso: 10 ene. 2020.

23.Sarrouf, E.B.; Souza S, R.; \& Gonçalves C, O. Mortalidad atribuible a gripe en los períodos preprogramas de inmunización y posprogramas de inmunización en Argentina: estudio ecológico (2002-2016). Rev Panam Salud Publica, 2019. [Internet]; 43:e15. Disponible en: http://iris.paho.org/xmlui/handle/123456789/49757 Acceso: 04 oct. 2019. 


\section{FIGURAS Y TABLAS}

Figura 1. Distribución del presupuesto invertido en SP por el MSPS-Col, Año 2016.

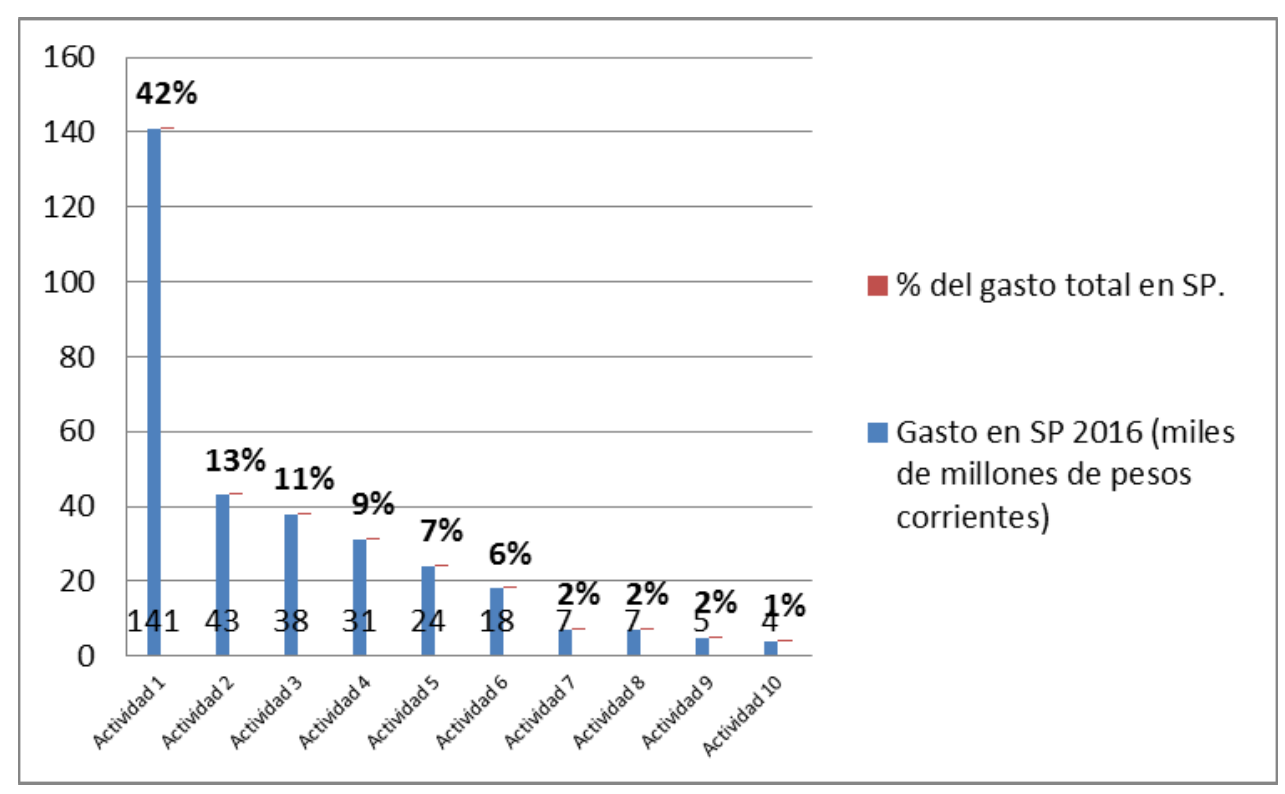

Figura 2: Distribución de la inversión presupuestal en actividades de Salud Pública (Departamentos de Colombia, 2017) *En miles de millones de pesos/año.

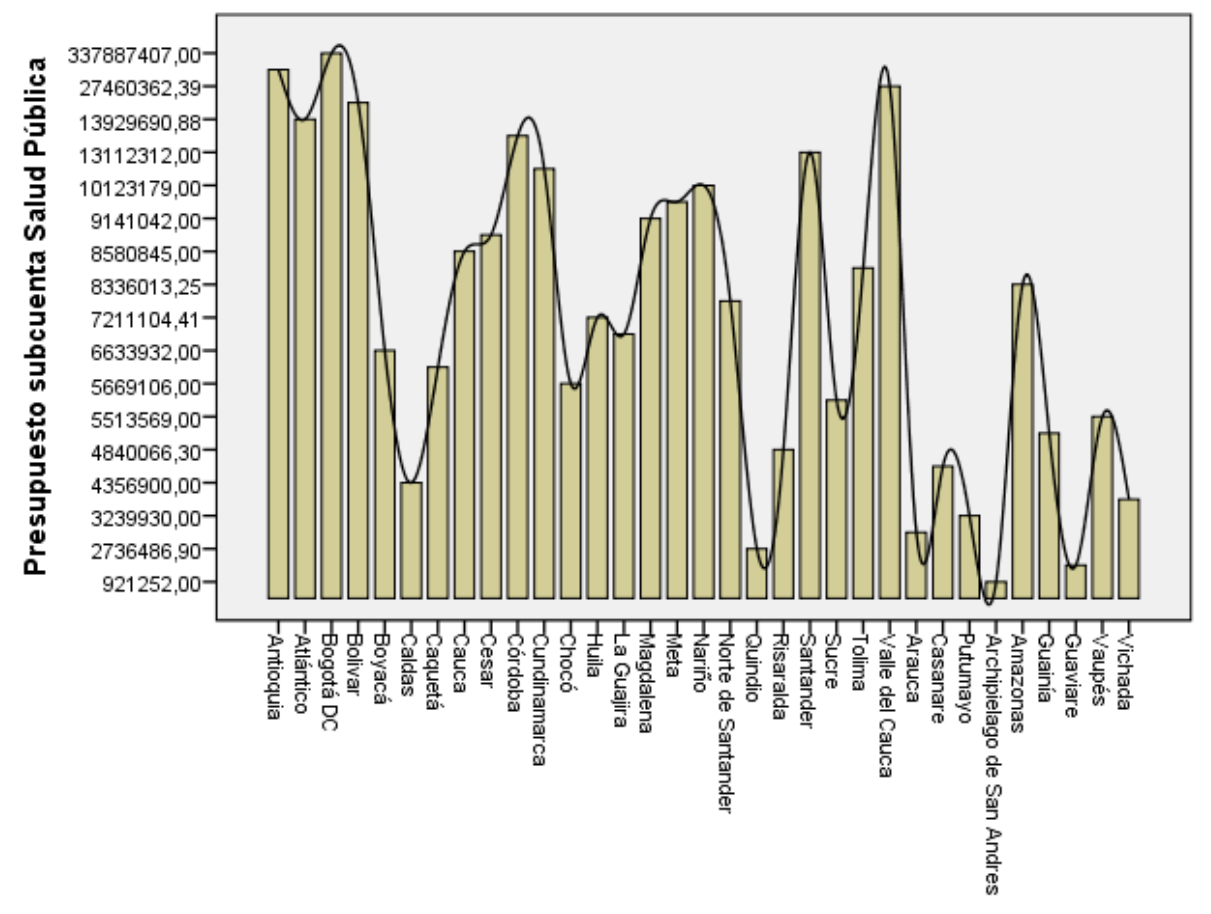

Departamento Colombia

Fuente: Elaboración propia a partir de los datos recopilados del Ministerio de Hacienda, Departamento Nacional de Planeación (DNP). 
Figura 3: Distribución de la Cobertura de Programas de inmunización y de las tasas de mortalidad asociada a IRA (Departamentos de Colombia, 2017) *\%.

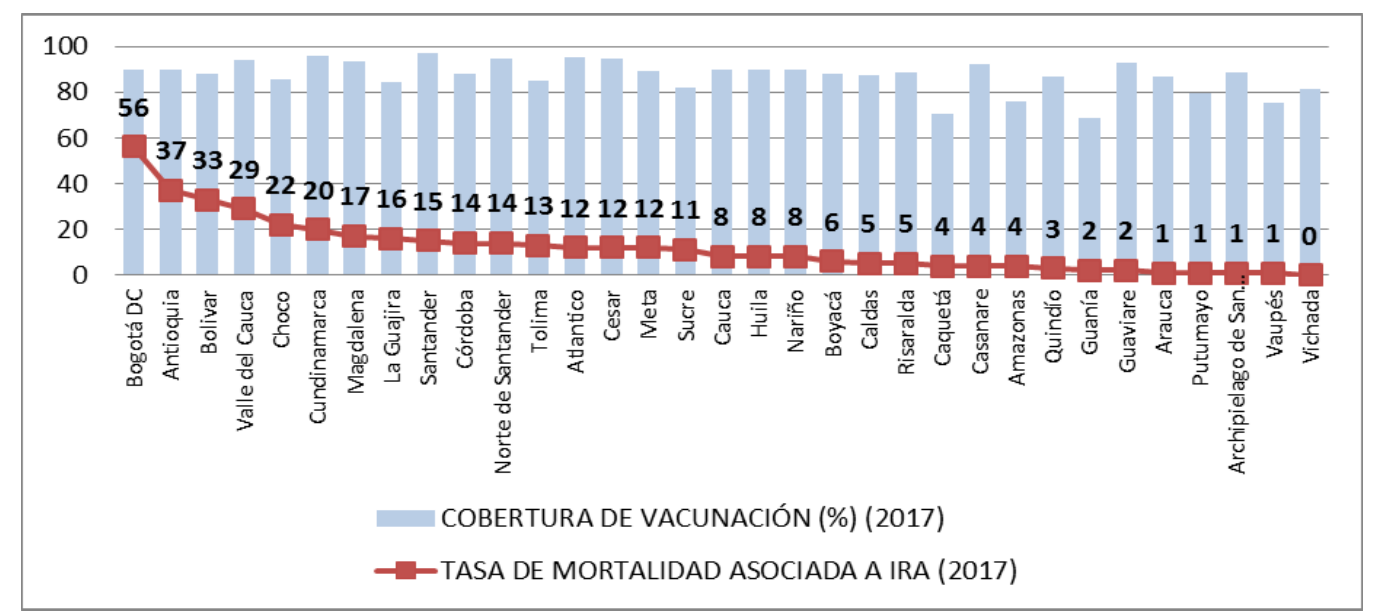

Fuente: Elaboración propia a partir de los datos recopilados del Ministerio de Hacienda, Departamento Nacional de Planeación (DNP).

Figura 4. Gráfico de dispersión para las variables Mortalidad por IRA y Cobertura de Programas de inmunización con Pentavalente Menor a 1 Año 3a. Dosis.

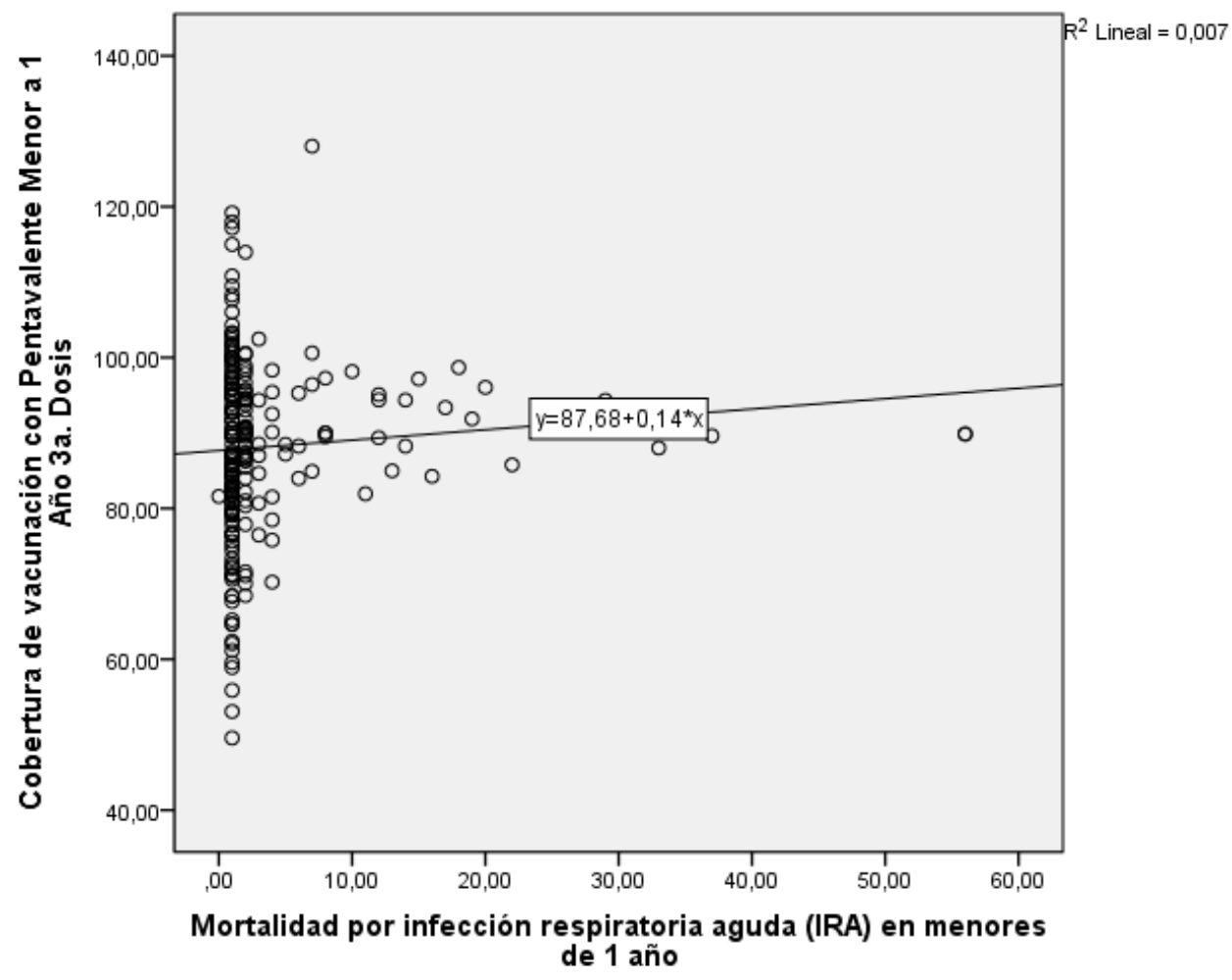

Fuente: Elaboración propia a partir de los datos recopilados del Ministerio de Hacienda, Departamento Nacional de Planeación (DNP). 
Figura 5. Comparación de las coberturas de programas de inmunización, el porcentaje del total del presupuesto ejecutado en Salud Pública, y la Tasa de Mortalidad asociada a IRA para Bogotá DC, Antioquia,

Valle del Cauca y Huila.

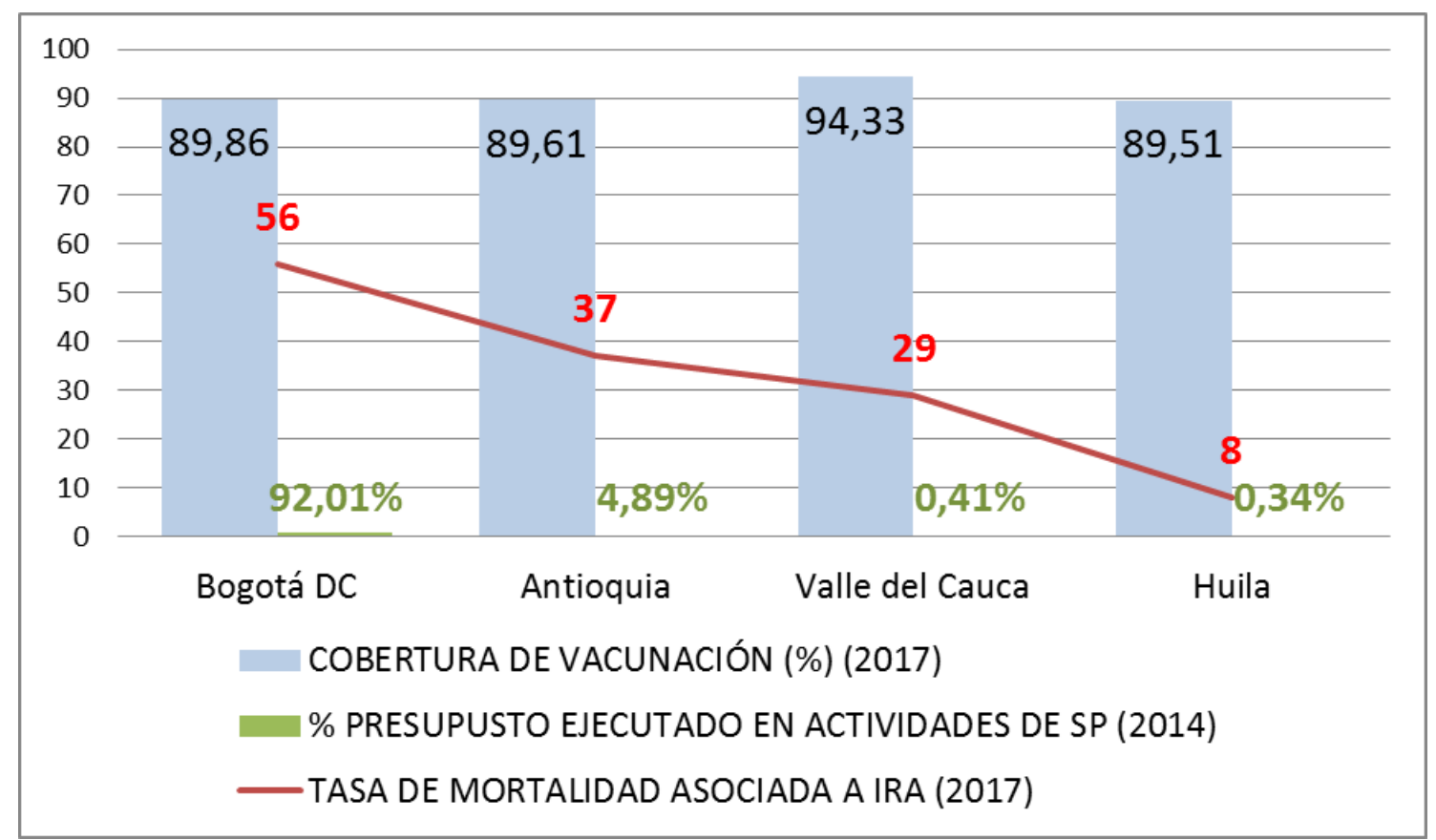

Fuente: Elaboración propia a partir de los datos recopilados del Ministerio de Hacienda, Departamento Nacional de Planeación (DNP).

Figura 6. Tasa de mortalidad por infección respiratoria aguda (IRA) en menores de 5 años - georreferenciado (Colombia 1998 - 2019).

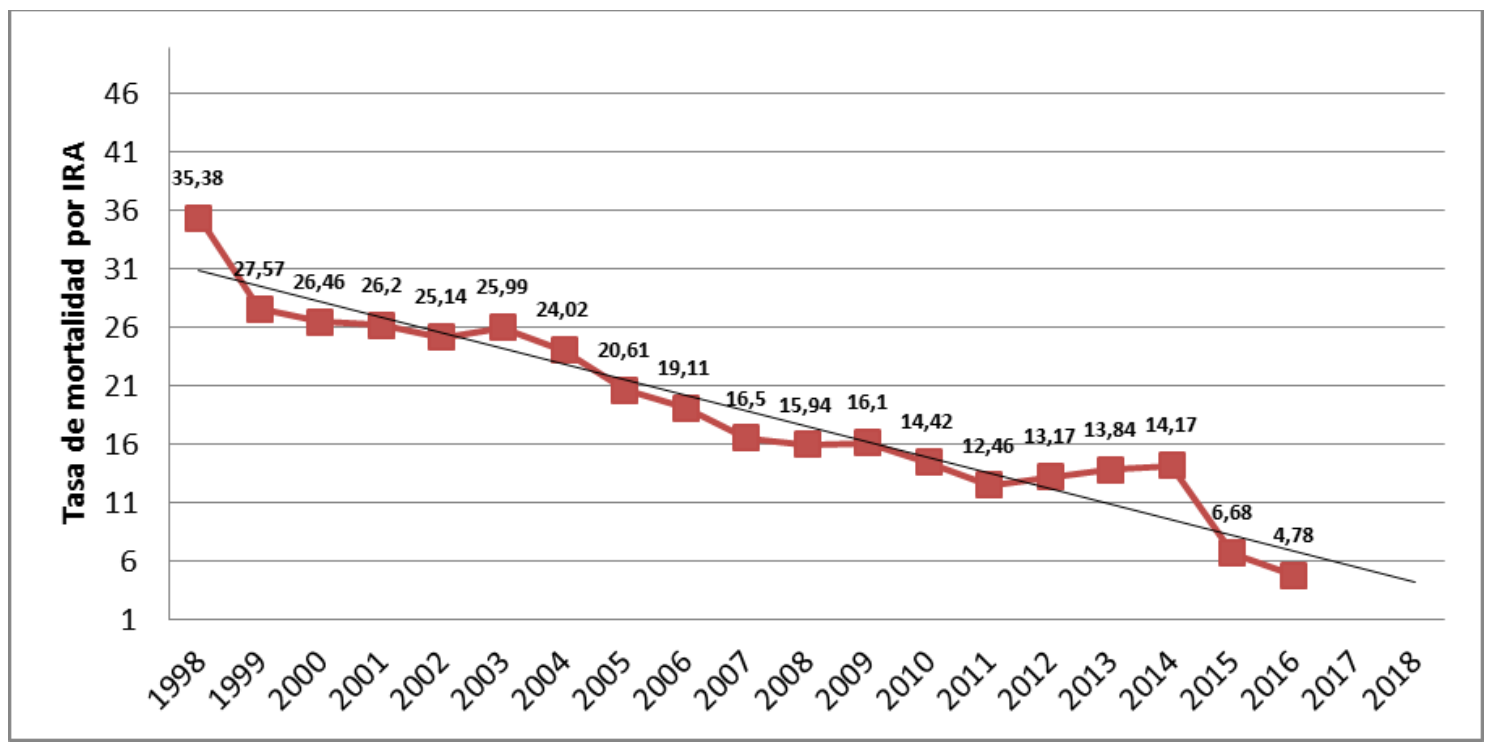

Fuente: Así Vamos en Salud (10). 
Daza-CAstilio - Mejía-Guatibonza - Pacheco Hernández GASTO EN SALUD PÚBLICA Y SU IMPACTO SOBRE LA MORTALIDAD POR INFECCIÓN RESPIRATORIA AGUDA

Tabla 1: Porcentajes de ejecución del presupuesto en SP y Coberturas de Inmunización con Pentavalente.

\begin{tabular}{|c|c|c|}
\hline DEPARTAMENTO & $\begin{array}{l}\text { EJECUCIÓN DEL } \\
\text { PRESUPUESTO (\%) }\end{array}$ & $\begin{array}{l}\text { COBERTURA DE } \\
\text { INMUNIZACIÓN (\%) }\end{array}$ \\
\hline Antioquia & $0,42 \%$ & $89,6 \% 1$ \\
\hline Atlántico & $0,17 \%$ & $95,09 \%$ \\
\hline Bogotá DC & $4,90 \%$ & $89,86 \%$ \\
\hline Bolívar & $0,18 \%$ & $88,02 \%$ \\
\hline Boyacá & $0,08 \%$ & $88,26 \%$ \\
\hline Caldas & $0,05 \%$ & $87,18 \%$ \\
\hline Caquetá & $0,07 \%$ & $70,23 \%$ \\
\hline Cauca & $0,11 \%$ & $89,98 \%$ \\
\hline Cesar & $0,11 \%$ & $94,38 \%$ \\
\hline Córdoba & $0,16 \%$ & $88,26 \%$ \\
\hline Cundinamarca & $0,15 \%$ & $96,04 \%$ \\
\hline Choco & $0,07 \%$ & $85,78 \%$ \\
\hline Huila & $92,21 \%$ & $89,51 \%$ \\
\hline La Guajira & $0,09 \%$ & $84,25 \%$ \\
\hline Magdalena & $0,11 \%$ & $93,36 \%$ \\
\hline Meta & $0,12 \%$ & $89,35 \%$ \\
\hline Nariño & $0,12 \%$ & $89,99 \%$ \\
\hline Norte de Santander & $0,10 \%$ & $94,36 \%$ \\
\hline Quindío & $0,03 \%$ & $87 \%$ \\
\hline Risaralda & $0,06 \%$ & $88,49 \%$ \\
\hline Santander & $0,16 \%$ & $97,16 \%$ \\
\hline Sucre & $0,07 \%$ & $81,94 \%$ \\
\hline Tolima & $0,10 \%$ & $84,98 \%$ \\
\hline Valle del Cauca & $0,34 \%$ & $94,33 \%$ \\
\hline Arauca & $0,04 \%$ & $86,96 \%$ \\
\hline Casanare & $0,05 \%$ & $92,51 \%$ \\
\hline Putumayo & $0,04 \%$ & $79,62 \%$ \\
\hline Archipiélago de San Andrés & $0,01 \%$ & $88,49 \%$ \\
\hline Amazonas & $0,10 \%$ & $75,79 \%$ \\
\hline Guaviare & $0,03 \%$ & $68,45 \%$ \\
\hline Vaupés & $0,07 \%$ & $93,02 \%$ \\
\hline Vichada & $0,04 \%$ & $75,07 \%$ \\
\hline Guaina & $0,06 \%$ & $81,59 \%$ \\
\hline
\end{tabular}

Fuente: Elaboración propia a partir de los datos recopilados del Ministerio de Hacienda, Departamento Nacional de Planeación (DNP). 